\title{
Percepción de recursos convencionales y no convencionales en áreas sujetas a estrés hídrico: el caso de Alicante ${ }^{1}$
}

\author{
Hug March², María Hernández ${ }^{3}$ y David Saurí4
}

\begin{abstract}
RESUMEN
Los denominados recursos hídricos convencionales se encuentran crecientemente y a nivel global sujetos a una confrontación social debido a sus impactos ambientales y socioeconómicos. En paralelo se están implementando con fuerza recursos alternativos como la desalación y la reutilización de aguas residuales depuradas. A pesar del gran potencial que pueden presentar, también pueden ser rechazados por la población, y en este sentido contamos con un conocimiento limitado acerca de cómo estos recursos alternativos son percibidos por la población. El presente artículo utiliza el caso de la provincia de Alicante, un área sujeta a un frágil equilibrio hídrico en la costa mediterránea española, para explorar la percepción ciudadana de distintas alternativas hídricas, con especial énfasis en la reutilización de aguas depuradas. Ello se ha llevado a cabo a través de una encuesta efectuada a una muestra de 450 residentes de diez municipios alicantinos. Los principales resultados obtenidos muestran que, la desalación, la estrategia principal Ilevada a cabo en España para sustituir a los trasvases, no goza de gran preferencia entre la población y es superada por las aguas pluviales y la reutilización de aguas regeneradas. La encuesta nos muestra que esta última alternativa, con un gran potencial de suministro, estaría ampliamente aceptada para usos que no impliquen contacto directo con el cuerpo humano.
\end{abstract}

Palabras clave: Recursos hídricos, aguas regeneradas, percepción, ámbitos urbanos y turísticos, España.

\begin{abstract}
The so-called conventional water resources are increasingly subject to social contestation due to their environmental and socio-economic impacts. In parallel, various alternative resources are being implemented, such as desalination and reuse of treated wastewater. Despite their great potential, we have a limited understanding of how these alternative resources are perceived by the population, particularly whether they will be accepted or not. This article presents a case study of the province of Alicante, an area along the Spanish Mediterranean coast with a delicate water balance, to explore public perceptions of various alternative water sources, with special emphasis on the reuse of treated wastewater. Herein we present a survey of 450 residents from ten municipalities within the region of Alicante. The main results show that, desalination; the main strategy carried out in Spain to replace dams, does not appeal to respondents and lags behind rainwater and the reuse of reclaimed water in terms of social preferences. The survey shows that water reuse would be widely accepted provided that it is used in forms that do not involve direct contact with the human body.
\end{abstract}

Key words: Water resources, treated wastewater, perception, urban and touristic areas, Spain.

\footnotetext{
1 Esta investigación ha sido financiada por la CICYT española (proyectos CSO2012-36997-C02-01 y CSO201236997-C02-02). El primer autor reconoce financiamiento del Ministerio de Economía y Competitividad de España (JCl-2011-10709) durante el período 2012-2014. Artículo recibido 24 de julio de 2014, aceptado el 15 de diciembre de 2014 y corregido el 15 de enero de 2015
}

2 Internet Interdisciplinary Institute, Universitat Oberta de Catalunya (España). E-mail: hmarch@uoc.edu

3 Instituto Interuniversitario de Geografía, Universidad de Alicante (España). E-mail: maria.hernandez@ua.es

4 Departamento de Geografía, Universidad Autónoma de Barcelona (España).E-mail: david.sauri@uab.cat 
La historia del abastecimiento de agua en zonas urbanas coincide muchas veces con la historia de la propia urbanización. A medida que el crecimiento de las ciudades ha ido avanzando, aumentan también las necesidades de agua, al tiempo que la disponibilidad de dicho recurso estimulaba a su vez la expansión urbana (Deutsch \& Gautheron, 2013; March, 2013; Melosi, 2000). En este proceso, las fuentes locales resultaban cada vez más insuficientes para saciar la sed de las ciudades y el agua debía ser transportada desde lugares más y más lejanos. Así, la historia de grandes capitales europeas, como París, Londres, Madrid y otras no se puede entender sin la continua búsqueda de recursos hídricos en lo que Erik Swyngedouw (2004) ha denominado la concatenación entre "flujos de agua y flujos de poder". Efectivamente, el control sobre los flujos de agua se ha identificado con el control sobre la ciudad, tal y como queda reflejado de manera magistral en Chinatown, el filme de Roman Polanski sobre el abastecimiento de agua a Los Ángeles durante la década de 1930.

Sin embargo, durante las últimas décadas, la aparentemente interminable búsqueda de nuevos recursos para satisfacer las demandas urbanas de agua empieza a mostrar signos de cambio. En primer lugar y por diversos motivos, esta demanda urbana de agua en muchas ciudades del mundo desarrollado parece estar disminuyendo a raíz de caídas más o menos pronunciadas en los consumos (March y Saurí, 2014; Gil et al., en prensa). En segundo lugar, en algunos casos como en el litoral mediterráneo de la Península Ibérica, grandes proyectos de desarrollo hídrico vinculados a un urbanismo primero en expansión y luego en caída libre no se han materializado, especialmente aquellos consistentes en la realización de trasvases intercuencas. En tercer lugar, los crecientes problemas en términos ambientales, sociales y económicos de soluciones convencionales como embalses y trasvases habrían motivado un replanteamiento general de estas soluciones, tal y como se puso de manifiesto en el Plan Hidrológico Nacional español de 2001 y la feroz oposición que suscitó su principal proyecto como era el trasvase del río Ebro (Saurí, 2003).

Precisamente a raíz de estos problemas, durante los últimos dos decenios han empe- zado a cobrar fuerza los denominados recursos no convencionales, especialmente la desalación (Olcina y Moltó, 2010). La desalación, en particular, se ha erigido como un recurso hídrico clave en los espacios áridos y semiáridos. En la cuenca mediterránea, por ejemplo, la desalación ya constituye la principal fuente de abastecimiento de muchos espacios insulares y en países como Israel se ha erigido también como fuente básica en el suministro de las ciudades (Feitelson \& Rosenthal, 2012). En España, si bien la primera desaladora fue construida en la isla de Lanzarote (Islas Canarias) en 1965, el mayor impulso a la utilización de aguas desaladas se vincula a la aprobación del programa AGUA (Actuaciones para la Gestión y la Utilización del Agua) a raíz de la derogación del Plan Hidrológico Nacional de 2001 (Rico, 2010; March et al., 2014). España ocupa la tercera posición mundial en capacidad de desalación instalada desde 2003 (Cajigas, 2012).

La apuesta por esta nueva fuente de suministro hídrico, resultado de la llegada al gobierno del Partido Socialista Obrero Español, se vincula al debate que desde mediados de la década de 1990 se establece entre aquellos que postulaban por la continuidad del denominado paradigma hidráulico y los que abogaban por la necesidad de un cambio en las políticas hidráulicas (Sauri y Del Moral, 2001; Del Moral, 2010); debate que se prolonga hasta la actualidad y que se ha materializado en tensiones más o menos larvadas entre partidos políticos defensores de unas $u$ otras propuestas y entre regiones cedentes y regiones receptoras de caudales a través de trasvases hídricos (Uche y Valero, 2001; Albiac et al., 2007). El programa AGUA planteaba una fuerte apuesta por la desalación al considerarla como la alternativa más adecuada para equilibrar los balances hídricos en las cuencas ya que, por un lado, evitaba las tensiones sociales que provocaban los trasvases $y$, por otro, porque suponía una medida eficaz contra la escasez de recursos en las regiones mediterráneas, acentuadas en periodos de sequía, al disponer de un recurso (el agua de mar) independiente de las condiciones climáticas.

El cambio en la política del agua se basaba, a priori, en la adopción de actuaciones 
concretas (plantas desaladoras) en cada territorio para garantizar la disponibilidad y la calidad del agua de estos ámbitos y reducir su vulnerabilidad ante episodios de sequía (Acuamed, 2013) y ante las previsiones de reducción de la pluviometría como consecuencia del cambio climático (Bates et al., 2008). Estos recursos no convencionales, sin embargo, poseen muchos rasgos en común con los convencionales centrados en embalses y trasvases. En primer lugar, en la mayor parte de los casos se trata de opciones tecnológicas a gran escala, que implican también grandes inversiones y que no quedan exentas de impactos económicos, sociales y ambientales. El programa AGUA tenía como actuación central la construcción de diversas plantas desaladoras a lo largo de la cuenca mediterránea española, con inversiones superiores a los 1.500 millones de euros (Acuamed, 2013). La desalación, sobre todo, ha sido objeto de crítica por sus costes energéticos que acentúan la dependencia energética de España (Estevan y García, 2007; Villar, 2014) y ambientales centrados en los vertido de salmueras y sus efectos en la posidonia oceánida (García y Ballesteros, 2001; Fernández-Torquemada y Sánchez-Lizaso, 2005). En segundo lugar, el desarrollo de estos recursos obedece a la misma lógica que los recursos convencionales, un discurso de seguir insistiendo sobre la oferta de agua en lugar de buscar alternativas más centradas en la gestión de la demanda. Finalmente, las relaciones de poder establecidas mediante el control de los flujos de agua de las grandes obras hidráulicas no se alterarían sustancialmente con estas nuevas alternativas (March, 2015), más proclives si cabe a procesos de privatización del suministro.

A estos rasgos comentados, hay que incorporar un nuevo elemento relacionado con el concepto de escasez. La utilización de la desalación ha significado en gran medida el fin de la escasez "física" de recursos en el litoral mediterráneo. Su uso permitiría generar recursos en abundancia. Sin embargo, diversos autores han empezado a referirse a una nuevo concepto, el de "escasez socioeconómica", que vendría definido por la dificultad de acceder a esos nuevos recursos por parte de los usuarios (Meerganz von Medeazza, 2005; Swyngedouw, 2013; March et al., 2014; March, 2015). El incremento del precio del agua como consecuencia de la incorporación de caudales procedentes de la desalación se ha traducido en un aumento del precio final pagado por el usuario. Un ejemplo paradigmático es el de la Mancomunidad de los Canales del Taibilla, una empresa pública que abastece en alta a más de dos millones de usuarios de las provincias de Alicante y Murcia. La diversificación de sus fuentes de suministro con la construcción de cuatro plantas desaladoras ha tenido repercusiones notorias sobre las tarifas del agua potable. Así, el precio del agua que suministra la Mancomunidad a los ayuntamientos y entidades ha crecido de $0,31 € / \mathrm{m}^{3}$ en 2005 , a 0,43 $€$ en 2007, a 0,544 € en 2009 y a 0,643 €/m³ en 2013. En apenas ocho años, las tarifas de agua potable servida en alta se han duplicado y ello en un contexto de aguda crisis económica (Gil et al., en prensa). Este incremento ha determinado la aparición de usuarios que tienen dificultades para poder acceder a este recurso o incluso que quedan excluidos del mismo, como ciertos usos agrarios y grupos sociales de bajo poder adquisitivo. Entre los primeros, excepto el cultivo de flores en invernadero, el resto de los aprovechamientos de regadío (hortalizas de invierno y cítricos) no pueden hacer frente a las nuevas tarifas del agua y mantener la viabilidad de sus explotaciones. Entre los segundos, el paulatino y constante incremento del agua en baja en los últimos años se ha traducido en el aumento de la morosidad y el fraude.

En conjunto, pues, la desalación sigue representando una alternativa con importantes puntos de contacto con el paradigma hidráulico convencional basado en las grandes obras gestionadas de una manera centralizada a partir de un enfoque "top-down" y que generalmente se justifican acudiendo a una escasez pretendidamente "natural" (Saurí y Del Moral, 2001). Sin embargo, la desalación también constituye hasta cierto punto una muestra de los cambios recientes en la gestión del agua, especialmente en lo que se refiere a una mayor presencia de intereses privados en esta gestión, también a nivel de abastecimientos en alta y para aquellos usos que, en principio, disponen de mayor capacidad económica (March, 2015). Por ejemplo, en Israel, las antiguas infraestructuras hidráulicas financiadas y gestionadas por el estado y con crecientes problemas de obsolescencia, deterioro, etc. han pasado a servir funda- 
mentalmente usos agrícolas mientras que las ciudades se abastecen mayoritariamente con agua desalada procedente de instalaciones privadas (Feitelson \& Rosenthal, 2012).

Junto a grandes obras y proyectos sean estos de fuentes convencionales o de fuentes no convencionales también cabe hablar de otras iniciativas que, si bien todavía muy incipientes y minoritarias, sí pueden ofrecer una alternativa futura nada desdeñable. Nos referimos a las múltiples iniciativas a escala local que intentan desarrollar proyectos de suministro de agua vinculados a recursos no convencionales como las aguas pluviales o las denominadas aguas grises. En estos casos, las actuaciones se hallan vinculadas muchas veces, al menos en España, a políticas ambientales a escala municipal como, por ejemplo, las denominadas Agendas 21 Locales cuyo objetivo es un uso más eficiente de los recursos en aras a promover el desarrollo sostenible del municipio. En la provincia de Barcelona y en materia de gestión del agua, la Diputación provincial ha impulsado el desarrollo de ordenanzas municipales de ahorro y conservación de agua en las que recursos alternativos como las aguas grises o las aguas pluviales se erigen como parte de los recursos globales de agua del municipio. Este tipo de alternativas se caracterizan por una serie de cambios muy notables con respecto a las soluciones anteriores. En primer lugar, se trata de proyectos a escala de vivienda unifamiliar (sobre todo para el caso de las aguas pluviales) o de bloque de pisos (escala más frecuente en el caso de las aguas "grises"). En segundo lugar, permiten una gestión y control de los recursos mucho más cercana al usuario ya que este es responsable o corresponsable del correcto funcionamiento de los sistemas. En el caso de las aguas pluviales, pueden representar también un cierto ahorro monetario para aquellos usuarios que tengan jardín y precisen de agua para regar (Domènech, 2011).

Finalmente, el caso de las aguas regeneradas, por sus características de producción centralizada, pero por sus usos diferenciados en función de la calidad del recurso, se situaría en una posición intermedia entre recursos no convencionales como la desalación y otros como las aguas grises o aguas pluviales. Por su parte, las aguas residuales depuradas continúan su avance en el proceso de cubrir un número de usos cada vez mayor. Si en una primera instancia, se utilizaban primordialmente para la recarga de acuíferos o el mantenimiento de ecosistemas húmedos, en la actualidad su uso se está extendiendo ya hacia el regadío a gran escala (con Israel otra vez como ejemplo) e incluso se vislumbran posibles opciones a nivel doméstico (Feitelson \& Rosenthal, 2012).

A pesar de un papel ciertamente todavía testimonial, estos nuevos recursos generados a escala local y, aún más, a escala de vivienda o bloque de viviendas representan un cambio cualitativo muy trascendente para el futuro de la gestión de agua urbana y más todavía por cuanto suelen ir asociados a otras estrategias de ahorro y conservación del recurso. Además, a menudo cuentan también con un apoyo importante por parte de distintos agentes sociales. En una encuesta efectuada a seis sectores vinculados con la gestión del agua y en la que se pedía la valoración de distintas alternativas no convencionales (desalación, reutilización de residuales, aguas grises y aguas pluviales), las aguas pluviales fueron el recurso evaluado más positivamente por parte de todos los participantes con excepción de las compañías de agua (Domènech et al., 2013a). Igualmente, en un análisis multicriterio de las mismas alternativas, que combinaba aspectos económicos, sociales y ambientales, las aguas pluviales registraron los valores más elevados exceptuando obviamente el, por otra parte muy crítico de seguridad en el suministro (Domènech et al., 2013a). A nivel de usuarios de estos recursos, una encuesta efectuada en el municipio de Sant Cugat (Área Metropolitana de Barcelona) señalaba una actitud generalmente favorable hacia el uso de aguas "grises" en bloques de pisos de construcción reciente a pesar de ciertos problemas en el mantenimiento de estos sistemas (Domènech \& Saurí, 2010).

Las encuestas anteriores no dejan de ser, sin embargo, aproximaciones un tanto parciales al estudio de la percepción de los recursos hídricos por parte de la ciudadanía en general. En este sentido, faltarían, al menos en nuestro contexto, estudios más globales sobre esta temática y que comparen de una manera sistemática la percepción de distintas opciones de suministro. Este tipo de estudios 
son más necesarios si cabe cuando existen controversias importantes en relación al futuro del suministro de agua en ciertas áreas. En el caso español, cabe recordar aquí la polémica suscitada por el proyecto de trasvase del río Ebro incluido en el Plan Hidrológico nacional de 2001 y también la controversia social, económica, política y ambiental que envuelve a la desalación. Ejemplos de dichos debates creados por grandes infraestructuras hídricas abundan a nivel global. Un buen ejemplo de ello es el propio informe que realizó la Comisión Mundial de Represas (2000). En América Latina son conocidos los impactos y los conflictos derivados de los megaproyectos hídricos (ver por ejemplo Boelens et al., 2012; Latta y Gómez, 2014). En otras regiones, como en Asia los megaproyectos hídricos, incluyendo presas y trasvases, también han generado contestación social (ver por ejemplo Domènech et al., 2013b o Nüsser, 2013). También es importante mencionar las controversias que ha generado la desalación en distintas áreas geográficas del planeta (March, 2015), ya que es una tecnología que se está abriendo a nuevos mercados como, por ejemplo, Latinoamérica o Asia.

En estos debates pocas veces se tuvo en cuenta la opinión de las personas potencialmente afectadas por estas alternativas. Sin entrar a discutir si la opinión pública sobre distintas alternativas de suministro de agua tenga que ser o no el principal criterio en la toma de decisiones al respecto, sí que creemos que resulta importante conocer y valorar la percepción de la ciudadanía. Más concretamente, es relevante estar al tanto de la percepción pública sobre los recursos denominados "no convencionales" debido al peso cada vez más notable que están adquiriendo en la oferta final de suministro de agua en algunas áreas, no solo de España, y su previsible crecimiento futuro dada la mayor vulnerabilidad de las opciones convencionales ante, por ejemplo, los retos planteados por el cambio climático.

El presente artículo tiene por tanto la finalidad de contribuir al debate sobre las distintas alternativas de suministro de agua en áreas caracterizadas por estrés hídrico mediante un análisis de la percepción ciudadana de estas alternativas tomando como caso de estudio un conjunto de municipios de la provincia de Alicante. El artículo está organizado de la manera siguiente. Después de esta introducción, se ofrece una presentación del área de estudio con un énfasis especial en la historia de los abastecimientos urbanos y turísticos, desde recursos locales como las aguas subterráneas hasta recursos proporcionados por trasvases intercuencas y más recientemente recursos no convencionales cuyo ejemplo más notorio sería la desalación. La tercera parte del trabajo está destinada a presentar el diseño y la metodología seguida para la elaboración de la encuesta entre una muestra significativa de la población de diez municipios alicantinos y a la descripción de los principales resultados obtenidos. En cuarto lugar se procederá a la discusión de dichos resultados y la relevancia de estos para el diseño de políticas públicas de gestión de agua en el futuro. Finalmente, en las conclusiones del trabajo se recalcará la importancia de los recursos no convencionales aunque también se señalará las importantes diferencias que se observan, tanto a nivel del recurso específico en sí (por ejemplo, el contraste entre agua desalada y agua regenerada) como a nivel de escala (soluciones a gran escala y soluciones a pequeña escala).

\section{Agua y urbanización en los núcleos urbanos y turísticos de Alicante}

La insuficiencia de recursos hídricos ha sido considerada tradicionalmente como uno de los principales condicionantes para el desarrollo socioeconómico de la provincia de Alicante, situada en la costa mediterránea española (Figura $N^{\circ} 1$ ). Las iniciativas orientadas a incrementar los recursos hídricos tanto para los usos agrícolas como para abastecimiento de agua potable han sido una constante a lo largo de la historia en el sureste peninsular (Olcina y Rico, 2006). Las transformaciones económicas, fuertemente vinculadas con el uso de recursos hídricos, que acontecen en estas tierras desde mediados siglo XX las podemos sintetizar cronológicamente y grosso modo de la siguiente manera: 1) el incremento de las áreas regadas y la introducción de cultivos con mayores demandas hídricas orientados al mercado; 2) el crecimiento de los núcleos urbanos con la llegada de población procedente de regiones 
próximas atraídas por la demanda de mano de obra unido al aumento de los módulos de consumo por parte de la población; y 3) la implantación y desarrollo de las actividades turístico-residenciales. Todos estos cambios acentuaron notablemente las tensiones entre recursos disponibles y demandas crecientes. La satisfacción de las demandas será una constante por parte de las iniciativas públicas, pero este proceso favorecerá, a su vez, la aparición de nuevas demandas y la necesidad de recurrir a nuevos recursos (subterráneos y trasvases en una primera etapa y a los no convencionales, posteriormente) y a la articulación de complejos sistemas de abastecimiento. La Mancomunidad de los Canales del Taibilla, uno de los mayores complejos hidráulicos a escala nacional para el abastecimiento a poblaciones y pieza clave en las trasformaciones territoriales registradas por estas provincias desde 1960, evidencia las interrelaciones que se establecen entre desarrollo urbano-turístico y la satisfacción de las demandas hídricas. El desarrollo urbanoturístico de la provincia de Alicante explica que el $50 \%$ de la población abastecida por la Mancomunidad se concentre en municipios costeros o de segunda línea (Gil y Rico, 2008). También el Consorcio de la Marina Baixa, aunque a menor escala, encapsula las relaciones que se establecen entre desarrollo urbano-turístico, la gestión mancomunada de los recursos hídricos y la colaboración entre usuarios urbanos y agrícolas.

Las relaciones que se establecen entre disponibilidad de agua y urbanización en los núcleos urbanos y turísticos del litoral alicantino reflejan claramente el proceso descrito. Hasta la irrupción de las actividades turísticas, el litoral de Alicante, si exceptuamos la capital provincial, se caracterizaba por el predominio de las actividades primarias (agricultura y pesca) que se complementaban con actividades de servicios e industriales en los enclaves donde existía una cierta tradición

Figura $\mathrm{N}^{0} 1$

Áreas de estudio en la provincia de Alicante, España

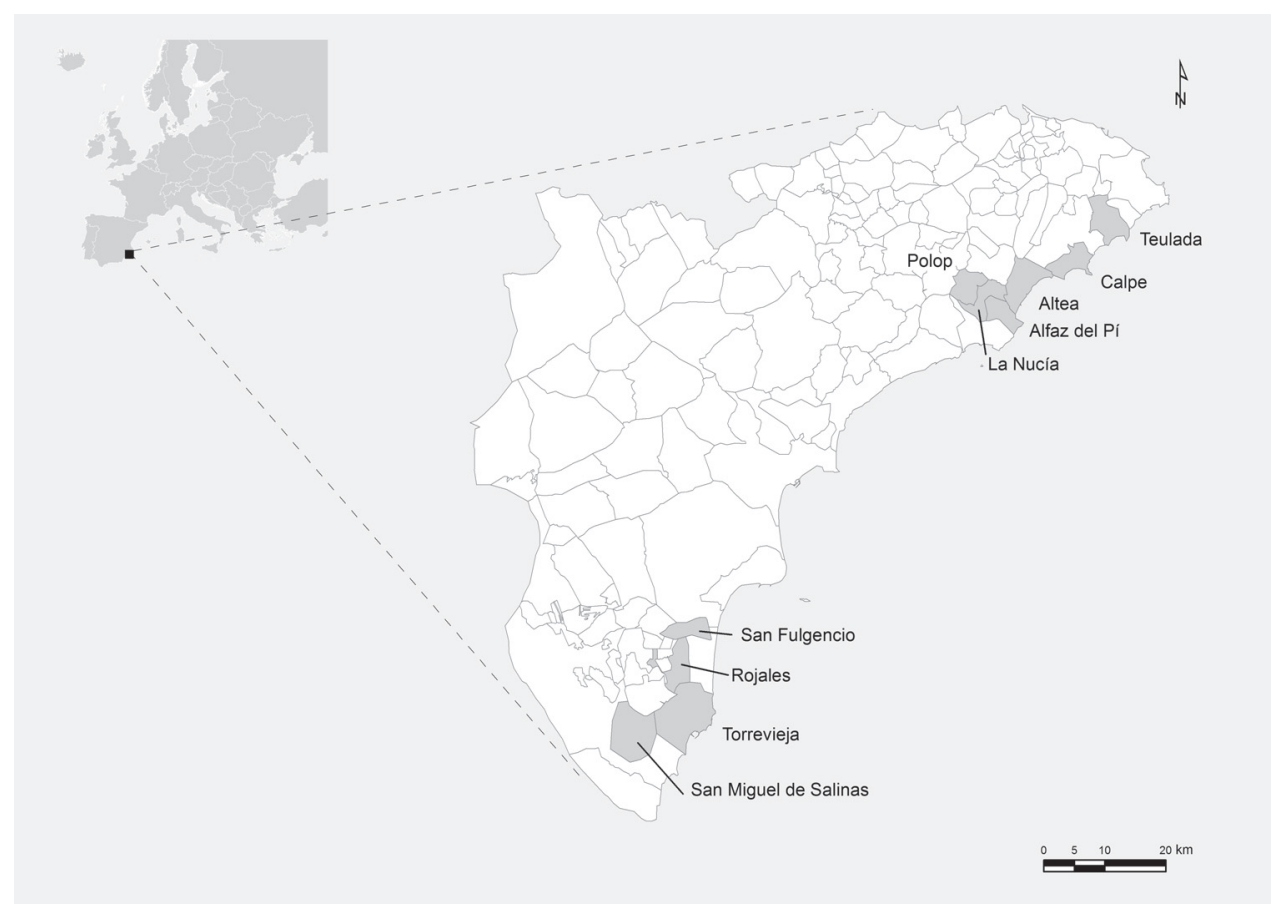

Fuente: Elaboración propia. 
como Torrevieja (Vera, 1987) o Villajoyosa. Salvo la capital provincial cuyos problemas de abastecimiento se habían resuelto en parte gracias a la creación de la Sociedad del Canal de la Huerta, que en 1907 logró la conexión de la cuenca artesiana del Alto Vinalopó y la ciudad de Alicante, mediante un canal de $55 \mathrm{~km}$, el resto seguía abasteciéndose mediante los escasos recursos locales (fuentes y manantiales, mayoritariamente).

La Mancomunidad de los Canales del Taibilla diseñada en 1927 en principio para dotar de agua potable a Cartagena y a su base naval, comprende en la actualidad más de $11.000 \mathrm{~km}^{2}$ y 79 municipios de las regiones de Murcia, Castilla-La Mancha y la Comunidad Valenciana de los cuales 34 corresponden a las comarcas alicantinas del Bajo Segura, Bajo Vinalopó y Campo de Alicante (incluyendo municipios como Elche, Orihuela, Torrevieja o Alicante). En total la Man- comunidad cubre una población en torno a los 2,5 millones de habitantes, que aumenta hasta los tres millones y medio en época estival (Cuadro $\mathrm{N}^{\circ} 1$ ). La importancia de la Mancomunidad a nivel regional se intensifica en época de sequía ya que este sistema puede suministrar caudales al Consorcio de Aguas de la Marina Baja, donde se incluyen municipios como Benidorm. El aumento de la población abastecida ha sido paralelo a una política de diversificación de fuentes de abastecimiento para hacer frente a las demandas crecientes. El análisis entre búsqueda de nuevos recursos hídricos y el desarrollo de las actividades económicas, básicamente urbano-turísticas en la provincia de Alicante pone de manifiesto las interrelaciones entre ambos elementos (Vera y Rico, 1995); relaciones que podemos sintetizar en cinco fases.

En una primera etapa, la principal fuente de abastecimiento fueron las aguas superfi-

\section{Cuadro $\mathrm{N}^{\circ} 1$}

Mancomunidad de los Canales del Taibilla. Evolución del número de habitantes abastecidos, volumen de agua suministrada y municipios mancomunados

\begin{tabular}{|l|r|r|r|}
\cline { 2 - 4 } \multicolumn{1}{c|}{} & $\mathrm{N}^{\circ}$ habitantes & \multicolumn{1}{c|}{$\mathrm{Hm}^{3}$} & $\mathrm{~N}^{\circ}$ municipios mancomunados \\
\hline 1950 & 125.000 & 5 & 15 \\
1960 & 830.000 & 61 & 26 \\
1970 & 1.190 .000 & 75 & 56 \\
1980 & 1.500 .000 & 130 & 71 \\
1990 & 1.600 .000 & 180 & 73 \\
2000 & 1.900 .000 & 195 & 79 \\
2004 & 2.300 .000 & 222 & 79 \\
2011 & 2.515 .003 & 214 & 79 \\
\hline
\end{tabular}

Fuente: Olcina y Rico, 2006: 352 y página web Mancomunidad de los Canales del Taibilla (http://www.mct.es)

ciales. El proyecto diseñado por el ingeniero José E. Ribera proponía captar las aguas del Taibilla, un afluente del Segura situado en su cabecera cuyo régimen de aportaciones era muy regular, y realizar una desviación de caudales de $2,5 \mathrm{~m}^{3} / \mathrm{seg}$. Como ya hemos comentado su objetivo inicial y principal era el abastecimiento de Cartagena y su base naval dada la penuria de recursos hídricos en esta área y abastecer así a los municipios del
Campo de Lorca, Murcia, Vegas del Segura y Alicante que no tenían otra posibilidad de suministro (Morales y Vera, 1989). Cuando en 1927 se creó la Mancomunidad de los Canales del Taibilla, 15 eran los municipios adscritos a este complejo, de los cuales 2 eran alicantinos (Orihuela y Elche). En 1950, se habían incorporado a este organismo un total de 23 nuevos municipios (17 de ellos alicantinos). A pesar del incremento de municipios, 
los recursos del río Taibilla eran suficientes para satisfacer las demandas crecientes. Esta afirmación se justifica por el hecho de que a mediados del siglo $\mathrm{XX}$, al igual que cuando se creó, la base económica mayoritaria era la agricultura y eran escasas las viviendas que contaban con agua corriente.

El proceso de adscripción de nuevos municipios (por ejemplo, Murcia en 1956 y Alicante y Elche en 1958) que se eleva a 56 en 1970, el aumento de la población abastecida (de 124.720 en 1950 a 1.178 .053 habitantes en 1970) (Gil y Rico, 2008), el incremento de los módulos de consumo por habitante y día gracias a las mejoras en los sistemas de abastecimiento y al incremento del nivel de vida, la creciente demanda industrial y finalmente el desarrollo turístico harán insuficiente esta fuente de abastecimiento hacia mediados de la década de 1960 cuando por primera vez las disponibilidades hídricas resultaron inferiores a las demandas. La estrategia que adoptará la Mancomunidad será la captación de nuevos recursos, situación que se mantendrá hasta la actualidad. Sin embargo el elemento diferenciador con el pasado será el recurso incorporado: primero se recurrió a las aguas del río Segura a la espera de la llegada de los caudales del trasvase del Tajo. El plan de Ampliación de los abastecimientos con recursos del Tajo al Segura fue aprobado por el Ministerio de Obras Públicas en 1971. Esta segunda etapa se prolonga hasta finales de la década de 1970 cuando de nuevo las demandas superan claramente a los recursos disponibles.

En la tercera fase, además de la incorporación de nuevos municipios (12 procedentes de la Vega Baja del Segura en 1979), el proceso fundamental que explica la insuficiencia de los recursos es el incremento de las demandas que experimentan los municipios litorales como consecuencia de la difusión de usos turísticos y, sobre todo, los residenciales en la década de 1960 y primeros años de la década siguiente. La solución a esta escasez vino de la llegada de caudales procedentes del Alto Tajo a través del trasvase Tajo-Segura en 1979. Estos nuevos volúmenes se traducen en un incremento significativo de los volúmenes distribuidos por la Mancomunidad, pasando de $75 \mathrm{hm}^{3}$ en 1970 a 130 en 1980 (Cuadro $\mathrm{N}^{\circ} 1$ ). Ello permitió la adscripción de nuevos municipios y, sobre todo, aseguró los caudales que, por un lado, garantizaron los usos existentes y a la vez espolearon la expansión de los usos residenciales, especialmente en el litoral sur de la provincia de Alicante durante las décadas de 1980 y 1990. El crecimiento continuo de las demandas hídricas, que se incrementan en $50 \mathrm{hm}^{3}$ más entre 1980 y 1990, se traduce a finales de la década de 1980 en la necesidad de recurrir a nuevos recursos hídricos una vez que se había alcanzado el tope de los dotacionales (110 hmªño) asignados a la Mancomunidad por la Regla de Explotación del Sistema de cabecera del río Tajo (Ley 52/1980, de 16 de octubre, "de regulación del régimen económico de la explotación del acueducto TajoSegura)" así como los caudales adicionales $\left(20 \mathrm{hm}^{3}\right)$ derivados de las menores pérdidas registradas en el transporte que las calculadas en el anteproyecto. Esta situación se vería agravada por varios procesos. En primer lugar, la disminución de las aportaciones del Taibilla a partir de 1987. En 2004, el volumen aportado se situaba en $43,2 \mathrm{hm}^{3}$, frente a los 50 hm³ $^{3}$ de 1996 y los 75 de 1990 (Olcina y Rico, 2006). En segundo lugar, los episodios de sequía, que de manera recurrente afectan a estas tierras, se traducen en una aminoración de los recursos disponibles procedentes del Alto Tajo y consiguientemente conllevan la necesidad de recurrir a recursos extraordinarios (como, por ejemplo, la apertura de pozos de sequía en el sinclinal de Calasparra). Otros caudales extraordinarios, que evitaron y/o minimizaron las restricciones al suministro fueron los procedentes de contratos de cesión de derechos de agua con regantes del río Tajo y del Segura (Gil y Rico, 2008).

La solución a esta insuficiencia, acentuada por el continuo incremento de las demandas (alrededor de un 10\% entre 2000 y 2005), va a proceder de la desalación, que abre la cuarta fase en nuestra periodización de la Mancomunidad. Esta opción fue contemplada por el Ministerio de Medio Ambiente con anterioridad a la derogación del trasvase del Ebro y su sustitución por el programa A.G.U.A. de 2004, expuesto en las secciones anteriores. Estos nuevos recursos, que complementan a los anteriores y forman parte de la política de diversificación de fuentes (Figura $N^{\circ} 2$ ), proceden de las plantas de desalación ubicadas en Alicante y en 
San Pedro del Pinatar. Estos recursos tienen como finalidad garantizar el abastecimiento de la demanda en aquellos periodos en los que, por motivos diversos (sequía, averías en el canal del trasvase, etc.), los recursos convencionales resultan insuficientes; así como reducir la presión existente sobre los recursos subterráneos y las trasferencias procedentes del Alto Tajo. Si bien su trascendencia en el volumen total suministrado por los Canales del Taibilla, se ha incrementado con el paso del tiempo, los flujos procedentes del Alto Tajo siguen siendo mayoritarios (alrededor del 60\%). En 2004, por ejemplo, los $222 \mathrm{hm}^{3}$ distribuidos por la Mancomunidad procedían del Taibilla $\left(43,2 \mathrm{hm}^{3}\right)$, del trasvase TajoSegura $\left(123 \mathrm{hm}^{3}\right)$, de recursos propios de las cuencas del Júcar y el Segura $\left(40 \mathrm{hm}^{3}\right)$ y los producidos por la desaladora del canal de Alicante $\left(16,8 \mathrm{hm}^{3}\right)$. El peso de esta fuente se incrementa con la entrada en funcionamiento de la desaladora de San Pedro del Pinatar. En 2005, ambas plantas aportaban unos $42 \mathrm{hm}^{3}$ cúbicos (Olcina y Rico, 2006); aportación teórica que se incrementa hasta los $93 \mathrm{hm}^{3}$ en 2011 con la entrada en funcionamiento de las 4 plantas desaladoras existentes: Alicante I (2003), Alicante II (2008), Antonio León Martínez Campos-San Pedro del Pinatar I (2005) y San Pedro del Pinatar II (2006) (Mancomunidad de los Canales del Taibilla, 2012). Las expectativas generadas por estos nuevos recursos unido a las políticas orientadas a la creación de suelo urbanizable por parte de los ayuntamientos y la fuerte demanda de viviendas (en ocasiones vinculada a procesos especulativos), entre otros factores, explican el notable boom urbanizador que registraron los municipios litorales y prelitorales de Alicante en el periodo comprendido entre 1998 y 2007 (Figura № 2). Esta expansión residencial vino acompañada de un ciclo de fuerte incremento de los consumos entre finales de la década de 1990 y primera década del siglo XXI y hasta los años 2007-2008.

La quinta y última fase viene definida por una contracción en los consumos, hecho que

Figura $\mathrm{N}^{\circ} 2$

Mancomunidad de los Canales del Taibilla. Evolución de la demanda $\left(\mathrm{HM}^{3}\right)$ según procedencia de los recursos.

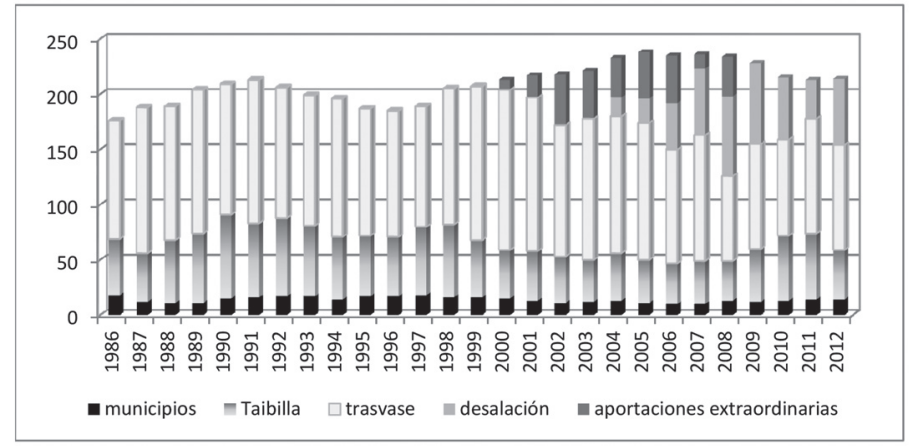

Fuente: Elaboración propia a partir de Mancomunidad de los Canales del Taibilla (2012: 29).

no había sucedido desde la creación de la Mancomunidad. La aminoración de las demandas, una situación que se repite en otras ciudades como Barcelona (March y Saurí, 2014; Gil et al., en prensa), responde a factores de tipo estructural y coyuntural. Entre los primeros cabe citar la mejora de la eficiencia en la red de distribución, la concienciación ambiental de los ciudadanos (consumo res- ponsable) que se traduce en una reducción de la dotación bruta por vivienda, el incremento de las tarifas y la implementación de medidas orientadas hacia una sostenibilidad territorial. La aprobación de la ley 4/2004 de "Ordenación del territorio y protección del paisaje" en su artículo 19 (uso sostenible del agua) hace mención a la necesidad de obtener un informe favorable del organismo 
de cuenca para la implantación de usos que supongan un incremento del consumo de agua; en otras palabras, un informe favorable acerca de "su disponibilidad y compatibilidad de dicho incremento con las previsiones de los planes hidrológicos, además de la no afectación o menoscabo a otros usos existentes legalmente implantados". Esta disponibilidad se puede justificar recurriendo a recursos procedentes de la desalación. Así la Confederación Hidrográfica del Segura accedió a tramitar peticiones de desaladoras privadas llevadas a cabo por diferentes constructoras para garantizar el agua para los nuevos complejos residenciales, incluidos en torno a una veintena de campos de golf (Ortuño et al., 2015). Sin embargo, desde finales de 2008 se han multiplicado los informes negativos sobre nuevos proyectos urbanos. Entre las causas de ello cabe mencionar la crisis económica que afecta a la economía española desde 2008 y el estallido de la burbuja inmobiliaria. Es este último un factor trascendental ya que ha supuesto una reducción notable en el número de viviendas visadas (Figura $N^{\circ} 3$ ). Esta notable contracción de la demanda se ha traducido en que no se hayan cumplido las proyecciones realizadas por la Mancomunidad de los Canales del Taibilla en los primeros años del siglo XXI y que cifraban las necesidades para el horizonte 2025 en unos 320-340 $\mathrm{hm}^{3}$ (Confederación Hidrográfica del Segura, 2007; Gil y Rico, 2008).

Las alternativas contempladas por la Mancomunidad de Canales del Taibilla, con

Figura $N^{\circ} 3$

Evolución de las viviendas visadas en la provincia de Alicante

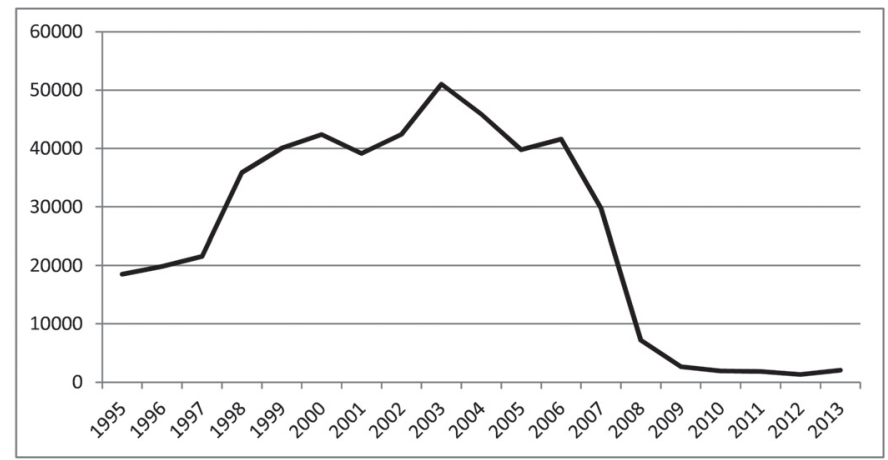

Fuente: elaboración propia a partir de Ministerio de Fomento (2012).

ser las de mayor peso en la matriz de abastecimientos urbanos y turísticos en Alicante, no resultan, sin embargo, las únicas posibles. Existen otros recursos no convencionales que también pueden satisfacer al menos parte de la demanda de agua pero cuya explotación es todavía muy incipiente o francamente inexistente como sería el caso de las aguas pluviales y de las aguas residuales depuradas (o regeneradas). En el primero de los casos, el aprovechamiento de aguas pluviales es muy escaso y únicamente cabe hablar de casos relativamente aislados como los depósitos de agua de tormenta en la ciudad de Alicante (sin un uso definido), mientras que las iniciativas a escala local son inexistentes en la práctica. Por lo que concierne a las aguas regeneradas es cierto que la provincia de Alicante y la Comunidad Valenciana en general constituyen una de las áreas españolas con un mayor uso aunque siempre con finalidades no domésticas (Olcina y Moltó, 2010). En este sentido ya existen experiencias en territorio alicantino sobre intercambio de aguas regeneradas por aguas superficiales, entre los usos agrícolas y los urbanos (ejemplo de Benidorm). Sin embargo el uso de este tipo de recurso para usos domésticos es aún muy limitado y desconocemos la percepción que de ello pueda tener la ciudadanía. 
Para que estas iniciativas puedan prosperar y ser aceptadas por la ciudadanía resulta importante conocer de antemano cuál es su valoración social, especialmente en relación a las alternativas convencionales. En el siguiente apartado presentamos el diseño, metodología y los resultados de la encuesta realizada en diez municipios de Alicante para tal efecto.

\section{Encuesta de percepción de alternativas de suministro de agua}

\section{Diseño y metodología de la encuesta}

Se realizaron 450 encuestas telefónicas en 10 municipios de la provincia de Alicante (Figura $\mathrm{N}^{\circ} 1$ ) entre el 19 y el 26 de octubre de 2012. Los municipios seleccionados cuentan con un predominio de un modelo suburbano $y$, por tanto, con un potencial mayor de aplicación de alternativas descentralizadas para usos exteriores domésticos (por ejemplo, riego de jardines). La muestra, con un error de $\pm 4,62 \%(p=.005)$, fue estratificada de acuerdo con la población de cada municipio en 2011 (Cuadro $\mathrm{N}^{\circ} 2$ )

A pesar que el objetivo principal de la encuesta era obtener datos sobre la percepción de los ciudadanos sobre recursos hídricos, también estimamos conveniente caracterizar sociodemográficamente la muestra y ver si existían determinantes sociodemográficos significativos en tales percepciones. Por este motivo dividimos la encuesta en dos bloques: uno sobre preguntas específicas sobre recursos hídricos y otro sobre la caracterización sociodemográfica del/de la encuestado/a.

En lo que concierne la percepción sobre recursos hídricos el bloque constaba de tres

Cuadro $\mathrm{N}^{\circ} 2$

Población y número de encuestas en los 10 municipios de la provincia de Alicante estudiados

\begin{tabular}{|l|r|r|}
\hline \multicolumn{1}{|c|}{ Municipio } & Población (2011) & Número de encuestas \\
\hline Alfàs del Pi & 21.332 & 38 \\
Altea & 24.006 & 42 \\
Calp & 29.909 & 53 \\
La Núcia & 18.225 & 32 \\
Polop & 4.294 & 8 \\
Rojales & 20.953 & 37 \\
San Fulgencio & 12.144 & 21 \\
San Miguel de Salinas & 8.057 & 14 \\
Teulada & 14.778 & 26 \\
Torrevieja & 101.091 & 179 \\
Total & 254.789 & 450 \\
\hline
\end{tabular}

Fuente: Elaboración propia.

apartados en los que se pedía a los encuestados que valorasen distintas afirmaciones mediante una escala likeart, que iba del 1 (en total desacuerdo) al 6 (en total acuerdo) (Cuadro $\mathrm{N}^{\circ} 3$ ). En el primer apartado, se presentaban distintas opciones de aumento del suministro, incluyendo opciones convencionales y alternativas, y se pedía la valoración de cada una. Los dos apartados siguientes se centraban en un recurso alternativo: las aguas regeneradas procedentes de la depuración. Nuestro interés específico por este recurso alternativo se justifica en primer lugar por una cierta experiencia en su uso en la Comunidad Valenciana, como ya hemos comentado anteriormente, y muy especialmente por su 
gran potencial para suplir agua con una calidad suficiente para muchos usos domésticos durante episodios de estrés hídrico. En este sentido se confrontaba la percepción de los encuestados con distintas afirmaciones sobre las implicaciones de distintos usos de este recurso en términos de salud, económicos y energéticos.
En lo que concierne a la caracterización de los encuestados, el segundo bloque de la encuesta contenía una serie de preguntas sobre la edad, el género, nivel de estudios, tamaño medio e ingresos de los hogares, tipología de la vivienda y la factura de agua.

Cuadro $\mathrm{N}^{\circ} 3$

Afirmaciones/preguntas sobre la percepción de recursos hídricos en el área de Alicante

\begin{tabular}{|c|c|c|}
\hline Pregunta/Afirmación & Opciones & Escala likeart \\
\hline $\begin{array}{l}\text { En el caso que fuera necesario } \\
\text { aumentar la cantidad de agua } \\
\text { disponible en su ciudad, va- } \\
\text { lore... }\end{array}$ & $\begin{array}{l}\text { i) Aumentar las aportaciones } \\
\text { del trasvase Tajo-Segura; ii) } \\
\text { realizar el trasvase del Ebro; } \\
\text { iii) incrementar la capacidad } \\
\text { de desalación; iv) aprovechar } \\
\text { el agua de lluvia; v) reutilizar } \\
\text { las aguas residuales una vez } \\
\text { depuradas (regeneradas). }\end{array}$ & $\begin{array}{l}1=\text { en total desacuerdo; } 6=\text { en } \\
\text { total acuerdo }\end{array}$ \\
\hline $\begin{array}{l}\text { En relación a la reutilización } \\
\text { del agua una vez depurada, } \\
\text { valore las afirmaciones si- } \\
\text { guientes... }\end{array}$ & $\begin{array}{l}\text { i) Puede suponer un riesgo } \\
\text { para la salud humana; ii) su } \\
\text { obtención implica un coste } \\
\text { económico elevado; iii) su } \\
\text { obtención requiere una impor- } \\
\text { tante cantidad de energía. }\end{array}$ & $\begin{array}{l}1=\text { en total desacuerdo; } 6=\text { en } \\
\text { total acuerdo }\end{array}$ \\
\hline $\begin{array}{l}\text { Valorar la adecuación de las } \\
\text { aguas residuales depuradas } \\
\text { o aguas regeneradas para los } \\
\text { siguientes usos... }\end{array}$ & $\begin{array}{l}\text { i) Riego de campos de golf y } \\
\text { otras instalaciones deportivas; } \\
\text { ii) riego agrícola; iii) limpieza } \\
\text { de calles y parques; iv) riego } \\
\text { de parques y otros espacios } \\
\text { públicos; v) riego de jardines } \\
\text { privados; vi) Ilenar piscinas; } \\
\text { vii) Ilenar la cisterna de los } \\
\text { inodoros domésticos; viii) la- } \\
\text { var la ropa; ix) ducharse o la- } \\
\text { varse las manos; x) lavarse los } \\
\text { dientes; xi) cocinar; xii) beber. }\end{array}$ & $\begin{array}{l}\text { ( } 1=\text { uso muy adecuado; } 6= \\
\text { uso nada adecuado) } \\
\text { NOTA: en este caso revertimos } \\
\text { el orden de la escala likeart } \\
\text { para enfatizar el cambio de } \\
\text { valoración (de acuerdo/des- } \\
\text { acuerdo a adecuado/no ade- } \\
\text { cuado) }\end{array}$ \\
\hline
\end{tabular}

Fuente: Elaboración propia.

\section{Caracterización sociodemográfica de la muestra}

El balance en términos de género entre los encuestados fue equilibrado (mujeres, $\mathrm{n}=234$; hombres, $\mathrm{n}=216$ ), mientras que la edad media de los encuestados era de 55 años. Se observó una presencia minoritaria de encuestados menores de 35 años (inferior al $10 \%$ ) y una importante representación del grupo de mayores de 64 años (34.4\%), probablemente debido a una presencia más asidua en el hogar durante el horario de realización de la encuesta. Por otro lado, el tamaño medio de los hogares encuestados fue de 2,75 personas, con un $10 \%$ de los encuestados viviendo solos $(n=49)$. Por otro lado, un $17 \%$ de los hogares tenía por lo menos un hijo/a 
menor de 10 años y alrededor de la mitad de la muestra contenía por lo menos una persona de más de 60 años. Por lo que respeta al nivel de estudios, el porcentaje era muy equilibrado entre los que tenían estudios básicos (25\%), educación primaria (25\%), educación secundaria $(30 \%)$ y educación universitaria $(20 \%)$. A nivel de ingresos, solo dos terceras partes de la muestra respondieron. La mayor parte de estos últimos (alrededor de un 90\%) reveló ingresos netos por hogar por debajo de los 30.000 euros anuales; y más concretamente un $64 \%$ declaró ingresos por hogar inferiores a los 18.000 euros anuales. En lo que concierne la tipología de vivienda, el 60\% de encuestados vivía en un piso mientras que el 40 por ciento restante lo hacía en una casa adosada o unifamiliar. Más de la mitad de las casas adosadas y unifamiliares disponían de jardín ( $n=104)$, aunque escasamente un 30\% de ellas disfrutaba de piscina. Finalmente se preguntaba también a cuánto ascendía la factura de agua de los encuestados; entre los que sabían tal información (n=330) más de la mitad reconocían pagar entre 51 y 100 euros por factura, mientras que una cuarta parte pagaban entre 26 y 50 euros; un 14\% más de cien euros y solo un $2 \%$ menos de 25 euros.

\section{Preferencia sobre las distintas fuentes de} suministro de agua

En el Cuadro $\mathrm{N}^{\circ} 4$ se pueden observar las preferencias de los encuestados para aumentar el suministro de agua en caso fuese necesario. La opción más valorada fue el aprovechamiento del agua de Iluvia, seguida del aumento de las aportaciones del trasvase Tajo-Segura, la realización del trasvase del Ebro, la reutilización de aguas residuales tratadas y finalmente el incremento de la capacidad de desalación.

Cuadro $\mathrm{N}^{\circ} 4$.

Preferencias para aumentar la cantidad de agua disponible si fuese necesario

\begin{tabular}{|c|c|c|c|c|c|c|}
\hline & $\begin{array}{c}\text { Aumentar las } \\
\text { aportaciones del } \\
\text { trasvase Tajo-Segura }\end{array}$ & $\begin{array}{c}\text { Realizar el } \\
\text { Trasvase del } \\
\text { Ebro }\end{array}$ & $\begin{array}{l}\text { Incrementar } \\
\text { la capacidad } \\
\text { de desalación }\end{array}$ & $\begin{array}{l}\text { Aprovechar el } \\
\text { agua de lluvia }\end{array}$ & $\begin{array}{c}\text { Reutilizar las aguas } \\
\text { residuales depuradas } \\
\text { (regeneradas) }\end{array}$ \\
\hline \multirow[t]{2}{*}{$\mathrm{N}$} & Válidos & 386 & 384 & 407 & 437 & 426 \\
\hline & Perdidos & 64 & 66 & 43 & 13 & 24 \\
\hline \multicolumn{2}{|c|}{$\begin{array}{l}\text { Media } \\
\text { (de menos a } \\
\text { más deseable) }\end{array}$} & 5,06 & 4,88 & 4,20 & 5,65 & 4,36 \\
\hline \multicolumn{2}{|c|}{$\begin{array}{l}\text { Desviación } \\
\text { típica }\end{array}$} & 1,51 & 1,675 & 1,971 & 1,058 & 1,938 \\
\hline \multicolumn{2}{|c|}{$\begin{array}{l}\text { Casos en total } \\
\text { desacuerdo } \\
(\mathrm{N})\end{array}$} & 30 & 39 & 83 & 14 & 74 \\
\hline \multicolumn{2}{|c|}{$\begin{array}{l}\text { Casos en total } \\
\text { acuerdo }(\mathrm{N})\end{array}$} & 232 & 222 & 164 & 374 & 205 \\
\hline \multicolumn{2}{|c|}{$\begin{array}{l}\text { Posición } \\
\text { deseabilidad }\end{array}$} & 2 & 3 & 5 & 1 & 4 \\
\hline
\end{tabular}

Fuente: Elaboración propia.

De los resultados de la encuesta se pueden hacer dos constataciones importantes. La primera es que todas las opciones recibieron altas puntuaciones, aunque, sin embargo, la desviación típica reflejaba opiniones más divididas para algunas opciones, como, por ejemplo, la desalación, con un alto número de opiniones "en total desacuerdo". Por el contrario, el aprovechamiento de agua de Iluvia presenta una desviación muy baja, con 
solo 14 casos "en total desacuerdo" y una inmensa mayoría $(n=374)$ en "total acuerdo".

La segunda constatación es que existe una diferencia importante en el número de valores perdidos (o en otras palabras respuestas en la forma "no sabe/no contesta") entre las distintas opciones. Como se puede ver en el Cuadro $N^{\circ} 4$ mientras las opciones de trasvase tiene un elevado número de valores perdidos, las opciones de agua de Iluvia y aguas regeneradas tienen un número más bajo de tales valores. Esto nos lleva a afirmar que los encuestados tienen una opinión más formada (ya sea positiva o negativa) sobre las tecnologías alternativas que sobre las convencionales. Podríamos aventurarnos a decir que esto se puede deber en parte a las polémicas y conflictos alrededor de las grandes infraestructuras hídricas en el levante español. En este sentido resulta factible pensar que una parte de los encuestados se abstuviera de valorar (ni positivamente ni negativamente) tales alternativas convencionales para evitar polemizar sobre el tema.

\section{Percepción sobre la reutilización de aguas regeneradas: riesgos e inconvenientes}

En el Cuadro $\mathrm{N}^{\circ}$ 5, se muestran los resultados sobre la percepción de los riesgos del uso de aguas depuradas regeneradas. Como ya hemos comentado anteriormente nuestro interés en conocer la percepción sobre los usos de este recurso viene dado por su potencial para constituirse como una fuente alternativa importante en periodos de estrés hídrico. Observamos que la mayor preocupación de los encuestados al respecto es la elevada cantidad de energía requerida en el proceso, seguido por el coste económico y, muy lejos, por el riesgo que puede suponer para la salud.

Sin embargo es importante mencionar que aunque de manera agregada el riesgo para la salud humana sea visto como el inconveniente menos problemático, también es cierto que es el que presenta una polarización más importante de las respuestas y una desviación estándar mayor, indicando la dispersión de las respuestas entre las múltiples categorías intermedias. En todo caso podemos constatar que las dos otras afirmaciones también presentan una desviación estándar importante. Con todo, quizá el dato más significativo sea, como ya se ha dicho, la menor aceptación de las aguas regeneradas desde una perspectiva energética y económica que desde una perspectiva de salud pública. Esta constatación adquiere una gran relevancia ya que uno de los motivos principales que se esgrime para no implementar tal alternativa es precisamente el presunto rechazo de la población por cuestiones de salud pública.

Cuadro $\mathrm{N}^{\circ} 5$

Percepción sobre los riesgos e inconvenientes reutilización aguas depuradas (regeneradas)

\begin{tabular}{|l|l|r|r|r|}
\hline \multicolumn{2}{|c|}{} & $\begin{array}{c}\text { Puede suponer un } \\
\text { riesgo para la salud } \\
\text { humana }\end{array}$ & $\begin{array}{c}\text { Su obtención implica } \\
\text { un coste económico } \\
\text { elevado }\end{array}$ & $\begin{array}{c}\text { Su obtención requiere } \\
\text { una importante cantidad } \\
\text { de energía }\end{array}$ \\
\hline $\mathrm{N}$ & Válidos & 395 & 304 & 301 \\
\cline { 2 - 5 } & Perdidos & 35 & 146 & 149 \\
\cline { 1 - 1 } $\begin{array}{l}\text { Media } \\
\text { Desviación típica }\end{array}$ & 2,039 & 4,35 & 4,51 \\
$\begin{array}{l}\text { Casos en total } \\
\text { desacuerdo (N) }\end{array}$ & 101 & 1,923 & 1,809 \\
Casos en total & 133 & 47 & 34 \\
acuerdo $(\mathrm{N})$ & & 146 & 150 \\
\hline
\end{tabular}

Fuente: Elaboración propia. 
Percepción sobre la reutilización de aguas depuradas: adecuación a distintos usos

En la tercera pregunta de la encuesta nos interesaba conocer cuáles eran los usos con los que el agua depurada regenerada podría encajar. En el Cuadro $N^{\circ} 6$, se presentan las valoraciones de todos los usos ordenadas de más a menos adecuadas según los encuestados.

Las distintas opciones quedan ordenadas de una manera muy coherente, con una muy alta aceptación de este recurso para usos exteriores y sobre todo públicos hasta una reticencia muy elevada al uso de estos recursos para funciones de higiene personal y, en especial, para consumo de boca. Estos resultados son muy interesantes si los comparamos con los de la pregunta anterior; re- cordemos que las respuestas a esta pregunta indicaban que los peligros para la salud eran menos prioritarios que el coste económico o energético. En contraste, para la pregunta que nos ocupa los encuestados presentan una reticencia muy elevada a utilizar este recurso para usos normalmente atribuidos al agua potable. Esta aparente contradicción podría explicarse por la percepción de que se trata de un recurso no problemático para ciertos usos (por ejemplo, el riego de jardines), pero también de un recurso para el que ni siquiera se asume un uso, como el de agua para beber o cocinar, que pueda suponer un riesgo para la salud.

Finalmente, se tiene que remarcar el bajo número de valores perdidos en todas las respuestas, demostrando que los encuestados tienen una opinión muy formada al respecto de esta cuestión.

\section{Cuadro $\mathrm{N}^{\circ} 6$}

Percepción sobre la adecuación de las aguas depuradas regeneradas para distintos usos

\begin{tabular}{|l|r|r|r|r|r|}
\hline Usos del agua regenerada & Válidos & Perdidos & Media & $\begin{array}{c}\text { Desviación } \\
\text { típica }\end{array}$ & $\begin{array}{r}\text { Muy adecuado (n) / } \\
\text { Nada adecuado (n) }\end{array}$ \\
\hline Limpieza de calles y parques & 438 & 12 & 1,63 & 1,465 & 348 / 35 \\
Riego de parques y otros & 437 & 13 & 1,66 & 1,471 & $340 / 33$ \\
espacios públicos & 431 & 19 & 1,89 & 1,768 & $326 / 59$ \\
Riego de campos de golf y & & & & & \\
otras instalaciones deportivas & 431 & 19 & 1,92 & 1,734 & $312 / 53$ \\
Riego de jardines privados & 436 & 14 & 1,95 & 1,814 & $322 / 63$ \\
Llenar la cisterna de los ino- & 432 & 18 & 2,41 & 2,007 & $259 / 84$ \\
doros domésticos & 420 & 30 & 3,38 & 2,182 & $152 / 142$ \\
Riego agrícola & 427 & 23 & 3,47 & 2,195 & $147 / 158$ \\
Llenar piscinas & 431 & 19 & 4,29 & 2,063 & 88 / 226 \\
Lavar la ropa & 432 & 18 & 4,78 & 1,938 & $67 / 293$ \\
Ducharse o lavarse las manos & 433 & 17 & 5,08 & 1,755 & $52 / 323$ \\
Lavarse los dientes & 434 & 16 & 5,21 & 1,702 & $51 / 345$ \\
Cocinar & & & & \\
Beber & & & \\
\hline
\end{tabular}

Fuente: Elaboración propia. 
Relación de las variables sociodemográficas con la preferencia por las distintas alternativas

Finalmente, se quiere complementar el análisis con una serie de tests estadísticos para inferir si existe cierta relación entre las variables sociodemográficas que caracterizan la muestra y las percepciones de los encuestados. En primer lugar, observamos un efecto del género en la preferencia de las distintas alternativas $(\mathrm{F}=6.329 ; \mathrm{p}=.096)$; pero únicamente en el caso del trasvase del Tajo-Segura, donde el sexo femenino presenta un grado mayor de aceptación de esta opción. Por otro lado, existe un efecto edad en lo que concierne a la percepción sobre el trasvase Tajo-Segura $(F=3,433 ; p=, 005)$, el trasvase del Ebro $(F=1,926 ; p=, 089)$ y el aprovechamiento de agua de lluvia $(\mathrm{F}=2,414$; $\mathrm{p}=, 036)$. En estos casos los grupos entre 45 y 55 y 55 y 64 años son lo que tienen una mejor opinión de estas opciones. Este hecho se puede relacionar con el argumento de que son estos grupos de edad los que han naturalizado de manera más importante el paradigma hidráulico característico de la política de aguas española durante el siglo XX. Por el contrario, podríamos argumentar que los grupos de menor edad, quizá más proclives a adoptar posiciones medioambientales, han experimentado el cuestionamiento de estos planteamientos tanto a nivel internacional y europeo así como más recientemente a nivel estatal.

En relación al nivel de estudios podemos ver un efecto significativo en la percepción de algunas alternativas. En este sentido cuanto más alto es el nivel de estudios menos de acuerdo se está con los trasvases del Tajo-Segura $(\mathrm{F}=6,818 ; \mathrm{p}=, 000)$ y del Ebro $(\mathrm{F}=9,926 ; \mathrm{p}=, 000)$ y más de acuerdo con la reutilización de aguas depuradas regeneradas $(\mathrm{F}=3,518 ; \mathrm{p}=, 015)$. Por otro lado, el efecto "ingresos" es similar al efecto "estudios" en lo que respeta a la deseabilidad de las distintas fuentes de suministro. Así, observamos una menor preferencia estadísticamente significativa por los trasvase del Tajo-Segura (excepto en el caso de las rentas más altas) $(\mathrm{F}=3,550 ; \mathrm{p}=, 015)$ y del Ebro $(\mathrm{F}=2,737$; $p=, 044)$ a medida que cambia el grupo de ingresos. Finalmente, en función del modelo residencial también vemos diferencias significativas en la percepción de la desalación $(\mathrm{F}=4,461 ; \mathrm{p}=, 035)$, con una mejor opinión proveniente de aquellos residentes en pisos más que en casas.

Relación de las variables sociodemográficas con la percepción sobre los riesgos y los usos de la reutilización de las aguas depuradas regeneradas

La variable que parece explicar más la diferencia de percepción sobre los riesgos e inconvenientes de este recurso alternativo son los ingresos. En este sentido, y de manera general, exceptuando las rentas más altas, podemos afirmar que a mayores rentas existe una menor problematización del uso de estos recursos tanto en términos de salud humana $(\mathrm{F}=2,317 ; \mathrm{p}=, 076)$ como de coste económico $(\mathrm{F}=2,618 ; \mathrm{p}=, 052)$. Existe un ligero efecto de la variable edad en la percepción que dicho recurso puede suponer un riesgo para la salud humana ( $F=2,036 ; p=, 073)$, con la población entre 36 y 45 con un mayor desacuerdo con tal afirmación. Ni el género, ni el nivel de estudios, ni la tipología de la vivienda dan ninguna diferencia estadísticamente significativa en la percepción de los problemas que conlleva este recurso. Finalmente, y dada la extensión de la tercera pregunta relativa a los usos, presentamos de manera muy sintética en el Cuadro $\mathrm{N}^{\circ} 7$ las variables que pueden generar diferencias significativas en la percepción de la idoneidad de este recurso alternativo para satisfacer distintos usos.

\section{Cuadro $\mathrm{N}^{\circ} 7$}

Percepción sobre la adecuación de las aguas depuradas regeneradas para distintos usos.

\begin{tabular}{|l|l|}
\hline & Efecto significativo \\
\hline $\begin{array}{l}\text { Limpieza de calles y parques } \\
\begin{array}{l}\text { Riego de parques y otros espacios } \\
\text { públicos }\end{array}\end{array}$ & Piso $(+)(\mathrm{F}=5,294 ; \mathrm{p}=, 022)$ \\
\hline
\end{tabular}




\begin{tabular}{|c|c|}
\hline & Efecto significativo \\
\hline $\begin{array}{l}\text { Riego de campos de golf y otras } \\
\text { instalaciones deportivas }\end{array}$ & Piso $(+)(F=3,541 ; p=, 061)$ \\
\hline Riego de jardines privados & Mujeres $(-)(F=5,891 ; p=, 016) ;$ Piso $(+)(F=5,618 ; p=, 018)$ \\
\hline $\begin{array}{l}\text { Llenar la cisterna de los inodoros } \\
\text { domésticos }\end{array}$ & - \\
\hline Riego agrícola & Mujeres $(-)(F=3,636 ; p==057)$ \\
\hline Llenar piscinas & Edad 45-55 (+) $(\mathrm{F}=2,215 ; \mathrm{p}=, 052)$ \\
\hline Lavar la ropa & $\begin{array}{l}\text { Edad 55-64 y más de } 64(-)(F=3,186 ; p=, 008) \text {; Estudios Bási- } \\
\cos (-)(F=3,353 ; p=, 019)\end{array}$ \\
\hline Ducharse o lavarse las manos & $\begin{array}{l}\text { Edad 55-64 y más de } 64(-)(F=3,727 ; p=, 003) \text {; Estudios Pri- } \\
\text { marios }(-)(F=3,657 ; p=, 013)\end{array}$ \\
\hline Lavarse los dientes & $\begin{array}{l}\text { Edad 45-55 (-) }(\mathrm{F}=4,841 ; \mathrm{p}=, 000) \text {; Estudios Primarios (-) } \\
(\mathrm{F}=2,218 ; \mathrm{p}=, 085)\end{array}$ \\
\hline Cocinar & Edad 45-55 (-) (F=2,702; $\mathrm{p}=, 020)$ \\
\hline Beber & - \\
\hline
\end{tabular}

Nota: (+) uso más adecuado; (-) uso menos adecuado Fuente: Elaboración propia.

\section{Conclusiones}

Este artículo ha presentado los principales resultados de una encuesta de percepción sobre distintas alternativas de suministro de agua en diez municipios de la provincia de Alicante. La demanda urbano-turística de agua en esta área se ha podido satisfacer con recursos convencionales superficiales (embalses, trasvases) y subterráneos así como, más recientemente, la desalación. Sin embargo, las dificultades económicas, sociales y ambientales de las grandes infraestructuras hidráulicas (incluyendo la desalación) y la sobreexplotación de buena parte de los acuíferos litorales hace necesaria la consideración de nuevas alternativas de suministro como, por ejemplo, las aguas pluviales o las aguas residuales depuradas, estas últimas objeto central de estudio en nuestra encuesta, dado su potencial para satisfacer una amplia gama de distintos usos. Sin embargo, se constata que estas alternativas únicamente tienen sentido si se acepta el aserto de que no todos los usos domésticos del agua precisan de una calidad óptima, hecho puede resultar particularmente significativo en un entorno residencial con una presencia importante de usos exteriores como es el caso de Alicante. En cualquier caso, una política pública que promueva la utilización de aguas residuales depuradas y de aguas pluviales requeriría un cierto conocimiento de la posible reacción ciudadana ante estos nuevos recursos, sobre todo en comparación a los recursos más convencionales. De ahí, el interés por explorar la percepción social de las distintas alternativas.

Por lo que se refiere a los resultados de la encuesta, en primer lugar habría que notar como el recurso de mayor aceptación sería el agua de lluvia, por delante de los trasvases (actuales y potenciales), de la reutilización de aguas residuales (regeneradas) y de la desalación. Este último caso es especialmente interesante por cuanto existe una cierta polarización en las opiniones, mientras que en el caso de los trasvases, hay cierta inhibición a la hora de responder. Nuestra hipótesis en ese caso es que las enormes controversias que han generado los trasvases pueden haber orientado a parte de la muestra a la no implicación ni en un sentido positivo ni en un sentido negativo, mientras que al menos parte de la oposición a la desalación puede deberse al alto coste económico de esta alternativa. En 
cambio, la reutilización de residuales y, sobre todo, el uso de pluviales quizá no motiven la controversia asociada con las otras fuentes y sean en este sentido más fáciles de aceptar.

En cuanto a las preguntas específicas sobre aguas regeneradas, sorprende que los encuestados observen mayores inconvenientes a nivel energético y económico que a nivel de posibles impactos sobre la salud humana. En cambio, cuando se pregunta por los usos que podría satisfacer este recurso, la preocupación por la salud humana aparece muy claramente manifestada ya que la mayor parte de los encuestados no utilizarían este recurso para el consumo de boca ni para la higiene personal. Para explicar esta contradicción, cabe suponer que los encuestados asumen implícitamente que las aguas regeneradas nunca se utilizarán para funciones que impliquen un contacto corporal pero que, en cambio, tienen potencialidades muy grandes para otros usos.

Los resultados de los análisis de inferencia estadística indican una cierta influencia de variables como la edad, los ingresos o los niveles educativos en las preferencias por algunas alternativas por encima de otras. Por ejemplo, unos mayores niveles educativos podrán contribuir a explicar la preferencia por la reutilización de aguas residuales y el mayor desacuerdo con alternativas como los trasvases. En cuanto a los costes y beneficios de la reutilización de residuales y con excepción de la rentas más altas, la variable "ingresos" parece ser la más indicada para explicar la mayor o menor consideración de los impactos económicos, energéticos y sobre la salud de estos recursos.

Finalmente, cabe señalar que la aceptación implícita de recursos no convencionales como la reutilización de aguas residuales se basa en gran parte en su potencial y no en experiencias ya contrastadas como sería el caso de otros recursos como el agua de los trasvases o de la desalación. En este caso, sería muy interesante poder someter a escrutinio público todas estas alternativas en ámbitos donde la coexistencia de pluviales y regeneradas con las alternativas más convencionales fuera más común y conocida por los usuarios. Sin embargo, y debido al descenso generalizado del consumo urbano de agua en esta y otras áreas de la península, no parece que los recursos alternativos se constituyan en recursos habituales para el ámbito doméstico, al menos a corto plazo.

\section{Referencias bibliográficas}

ACUAMED. La desalación en España. Sostenibilidad para zonas vulnerables. Madrid: Ministerio de Agricultura, Alimentación y Medio Ambiente, 2013.

ALBIAC, J.; HANEMANN, M.; CALATRAVA, J.; UCHE, J. \& TAPIA, J. The rise and fall of the Ebro Water transfer. Natural Resources Journal, 2007, Vol. 3, N46, p. 727-757,

BATES, B.; KUNDZWWICZ, Z.W.; WU, S. \& PALUTIKOF, J. (editors). Climate change and Water. Technical paper of the intergovernmental Panel on Climate Change. Ginebra: IPCC secretariat, 2008.

BOELENS, R.; DUARTE, B.; MANOSALVAS, R.; MENA, P. \& ROA AVENDAÑO, T. Contested Territories: Water Rights and the Struggles over Indigenous Livelihoods. The International Indigenous Policy Journal, 2012, Vol. 3, $\mathrm{N}^{\circ}$ 3. Disponible en Internet: http:// ir.lib.uwo.ca/iipj/vol3/iss3/5

CAJIGAS DELGADO, A. Jornada Iberoamérica: acción para el agua. Oportunidades de Negocio en el sector del agua. En: Congreso Nacional del Medio Ambiente (Conama), 26-30 noviembre 2012, Madrid.

COMISIÓN MUNDIAL DE REPRESAS. Represas y Desarrollo: Un Nuevo Marco para la Toma de decisiones. Ciudad del Cabo: Comisión Mundial de Represas, 2000.

CONFEDERACIÓN HIDROGRÁFICA DEL SEGURA. Plan de Actuación en Situaciones de Alerta y Eventual Sequía. Anejo 3. Demandas y Usos Consuntivos del Agua. Murcia: CHS, 2007.

DEL MORAL, L. The Hydraulic Paradigm and Production of a New Geography in Spain: Origins and Historical Evolution Between the Sixteenth and the Twentieth Centuries. In: Rivers and Society: From Early Civilizations to Moderm Times. Londres: I.B. Tauris, 2010, p. 440-462. 
DEUTSCH, J.C. \& GAUTHERON, I. (editors). Eaux pour la ville eaux des villes. Eugène Belgrand XIXe-XXIe siècle. Paris: Presses des Ponts, 2013.

DOMENECH, L. Rethinking water management: From centralised to decentralised water supply and sanitation methods. Documents d'Anàlisi Geogràfica, 2011, Vol. 57, Nº 2, p. 293-310.

DOMÈNECH, L. \& SAURI, D. Sociotechnical transitions in water scarcity contexts: Public acceptances of greywater reuse technologies in the Metropolitan Area of Barcelona. Resources, Conservation and Recycling, 2010, Vol. 55, № 1, p. 53-62.

DOMÈNECH, L.; MARCH, H. \& SAURÍ, D. Degrowth initiatives in the urban water sector? A Social Multi-criteria Evaluation of non-conventional water alternatives in Metropolitan Barcelona. Journal of Cleaner Production, 2013a, No38, p. 44-55

DOMÈNECH, L.; MARCH, H. \& SAURÍ, D. Contesting large-scale supply projects at both ends of the pipe in Kathmandu and Melamchi Valleys, Nepal. Geoforum, 2013b, Vol. 47 , p. 22-31.

ESTEVAN, A. \& GARCÍA, M. El consumo de energía en la desalación de agua de mar por ósmosis inversa: situación actual y perspectivas. Ingeniería Civil, 2007, No 48, p. 113-121.

FEITELSON, E. \& ROSENTHAL, G. Desalination, space and power. The ramifications of Israel's changing water geography. Geoforum, 2012, Vol. 43, p. 272-284.

FERNÁNDEZ-TORQUEMADA, Y. \& SÁNCHEZ-LIZASO, J. Effects of salinity on leaf growth and survival of the Mediterranean seagrass Posidonia oceanic (L) Delile. Journal of experimental marine biology and ecology, 2005, N³20, p. 557-63.

GARCÍA, E. y BALLESTEROS, E. El impacto de las plantas desalinizadoras sobre el medio marino: la salmuera en las comunidades bentónicas mediterráneas. En: Conferencia Internacional El Plan Hidrológico Nacional y la gestión sostenible del agua. Aspectos medioambientales, reuitilización y desalación. Zaragoza, 2001.

GIL, A. y RICO, A.M. Políticas del agua II. Mejora y ampliación de los riegos de Levante. Murcia: Esamur, 2008.

GIL, A.; HERNÁNDEZ, M.; MOROTE, A.F.; RICO, A.M.; SAURÍ, D. \& MARCH, H.: Causes of the tendencies of the water consumption by domestic use and big subscribers between 2007-2013, in the city of Alicante and the Metropolitan Area of Barcelona. Alicante: Universidad de Alicante (en prensa).

LATTA, A. y GÓMEZ, A. Agua y megaproyectos en Latinoamérica: Una introducción. European Review of Latin American and Caribbean Studies/Revista Europea de Estudios Latinoamericanos y del Caribe, 2014, № 97, p. 51-54.

MANCOMUNIDAD DE LOS CANALES DEL TAIBILLA. El organismo y su evolución histórica, 2012. Disponible en Internet: http:// mct.es/files/Biblioteca/Organismo\%20y\%20 su\%20evolucion/Organismo_y_su_evolucin_historica.pdf

MARCH, H. Taming, controlling and metabolizing flows: Water and the urbanization process of Barcelona and Madrid (18502012). European Urban and Regional Studies, 2013, doi: 10.1177/0969776412474665

MARCH, H. The politics, geography and economics of desalination: a critical review. WIREs Water, 2015, Vol. 2, No 3, p. 231-243.

MARCH, H.; SAURI, D. \& RICO-AMORÓS, A.M. The end of scarsity? Water desalination as the new cornucopia for the Mediterranean Spain. Journal of Hidrology, 2014, $\mathrm{N}^{\circ}$ 519(C), p. 2642-2651.

MARCH, H. y SAURÍ, D. ¿Qué sostenibilidad hídrica? Una interpretación crítica del descenso reciente del consumo doméstico de agua en Barcelona. Waterlat-Gobocit Network Working papers, 2014, Vol. 1, № 2, p. 27-43.

MELOSI, M. The Sanitary City. Urban infrastructure in America from Colonial times to the present. Baltimore: Johns Hopkins University Press, 2000. 
MEERGANZ VON MEDEAZZA, G.L. Direct and socially-induced environmental impacts of desalination. Desalination, 2005, Vol. $185, \mathrm{~N}^{\circ} 1-3$, p. $57-70$.

MINISTERIO DE FOMENTO. $V i$ viendas visadas. 2012. Disponible en Internet: http://www.fomento.gob.es/ $\mathrm{BE} /$ ? nivel $=2$ \&orden $=09000000$

MORALES, A. y VERA, J.F. La Mancomunidad de los Canales del Taibilla. Madrid: Academia Alfonso X El Sabio, 1989.

NÜSSER, M. (editors). Large Dams in Asia. Contested Environments between Technological Hydroscapes and Social Resistance. Heidelberg: Springer, 2013.

OLCINA, J. y RICO, A.M. La Mancomunidad de los Canales del Taibilla: un ejemplo de cooperación municipal surgido de una necesidad. En: ROMERO, J. y FARINÓS, J. (editores). Gobernanza territorial en España. Claroscuros de un proceso a partir del estudio de casos. Valencia: Publicaciones de la Universidad de Valencia, 2006, p. 347-374.

OLCINA, J. y MOLTÓ, E. Recursos de agua no convencionales en España. Estado de la cuestión. Investigaciones Geográficas, 2010, N51, p. 131-163.

ORTUÑO, A.; HERNÁNDEZ, M. \& ClVERA, S. Golf course irrigation and selfsufficiency water in southern Spain. Land use policy, 2015, № 44, p. 10-18.

RICO, A.M. Plan Hidrológico Nacional y Programa A.G.U.A. Repercusión en las regiones de Murcia y Valencia. Investigaciones Geográficas, 2010, № 51, p. 235-267.
SAURÍ, D. El transvassament de l'Ebre: Recursos hídrics, identitat i "equilibri territorial". In: NEL·LO, O. (editors). Aquí, no!. Els Conflictes territorials a Catalunya. Barcelona: Empuries, 2003, p. 252-273.

SAURI, D. y DEL MORAL, L. Recent development in Spanish water policy. Alternatives and conflicts at the end of the hydraulic age. Geoforum, 2001, Vol. 32, p. 351-362.

SWYNGEDOUW, E. Social Power and the urbanization of water. Oxford: Oxford University Press, 2004.

SWYNGEDOUW, E. Into the sea: desalination as hydro-social fix in Spain. Annals of the Association of American Geographers, 2013, Vol. 103, N² 2, p. 261-270.

UCHE, J. y VALERO, A. ¿Hasta qué punto es alternativa la desalación? Zaragoza: Universidad de Zaragoza, Centro de Investigación y Recursos Energéticos (CIRCEO), 2001.

VERA REBOLLO, J.F. Turismo y urbanización en el litoral alicantino. Alicante: Instituto de Estudios Juan Gil-Albert, 1987.

VERA REBOLLO, J.F. y RICO AMORÓS, A.M. Los sistemas de abastecimiento de agua potable en un espacio turístico y residencial: la Costa Blanca. En: BRU RONDA, C. y SANTAFÉ MARTínEZ, J. (editores). Agua y Espacios de Ocio. Alicante: Universidad Internacional Menéndez Pelayo y Fundación CAM, 1995, p. 105-150.

VILLAR, A. El coste energético de la desalinización en el programa A.G.U.A., Investigaciones Geográficas, 2014, № 62, p. 101-112. 